\title{
Pruritic breast mass with palpable lymph nodes in a male patient: a case report
}

\author{
Quanyi Long, Yuan Fan, Jia Zhang, Hongjiang Li, Qing Lv \\ Department of Breast Surgery, West China Hospital, Sichuan University, Chengdu, China \\ Correspondence to: Hongjiang Li; Qing Lv. Department of Breast Surgery, West China Hospital, Sichuan University, Chengdu, China. \\ Email: lihongjiang@sohu.com; lqlq1963@163.com.
}

\begin{abstract}
This paper presents a case study of a 78-year-old male patient who presented with exacerbated skin redness and edema on the left chest wall, especially on the left breast, and who had been suffering from associated pruritus for 6 months. The patient also presented with enlarged ipsilateral axillary lymph nodes that were suspected to be carcinomas after a preliminary ultrasound and enhanced computerized tomography (CT) examination were performed. To examine these symptoms, an ultrasound-guided core biopsy and a chronic inflammatory test were also performed. The results of the excision biopsy and the immunohistochemistry test of the left breast and ipsilateral lymph node revealed no signs of cancer in this patient. Finally, combined with his medical history, the laboratory tests and pathology results, the patient was diagnosed with plasma cell mastitis (PCM) after another suspicious lesion (e.g., inflammatory breast cancer, etc.) was excluded. PCM is a kind of benign lesion of the breast with an unclear etiology. It usually affects non-pregnant and non-lactational females, who display clinical symptoms that are often similar to those of inflammatory breast cancer (IBC), the main manifestations were erythema and edema on the chest wall. To date, there is no standardized clinical treatment strategy or management approach for PCM.
\end{abstract}

Keywords: Lymph node; plasma cell mastitis (PCM); inflammatory breast cancer (IBC)

Submitted Dec 21, 2020. Accepted for publication Feb 10, 2021.

doi: $10.21037 / g s-21-40$

View this article at: http://dx.doi.org/10.21037/gs-21-40

\section{Introduction}

Many of the pathological processes of breast diseases (for both benign and malignant lesions) in males are the same as those in females. Breast cancer in males accounts for only approximately $0.7 \%$ of all diagnosed breast cancers, but often affects males worse than females due to delays in diagnosis (1). Thus, it is essential that patients are diagnosed as early as possible. In males, the peak age of incidence for breast cancer ranges from 60 to 70 years. Inflammatory breast cancer (IBC) is a rapidly progressing malignant tumor that requires clinicians' close attention. Plasma cell mastitis (PCM) is a kind of inflammatory disease that often occurs in non-lactational and nonmenopausal women. The etiology and pathogenesis of PCM are unknown. Multifarious factors, such as a hormonal imbalance, smoking, and autoimmunity, are considered possible causes of the disease. The principal clinical manifestations of PCM are severe local pain and swelling accompanied by nipple discharge (2). While the histopathological hallmarks of PCM include the infiltration of abundant plasma cells, lymphocytes and macrophages around the mammary duct (2). We present the following article in accordance with the CARE reporting checklist (available at http://dx.doi.org/10.21037/gs-21-40).

\section{Case presentation}

A 78-year-old man was admitted to our clinic with a 6-month history of progressive skin redness and edema on the left chest wall and associated pruritus. The patient also complained of enlarging ipsilateral axillary lymph nodes. The patient had been diagnosed with diabetes and hypertension some 8 months earlier. The patient did not 


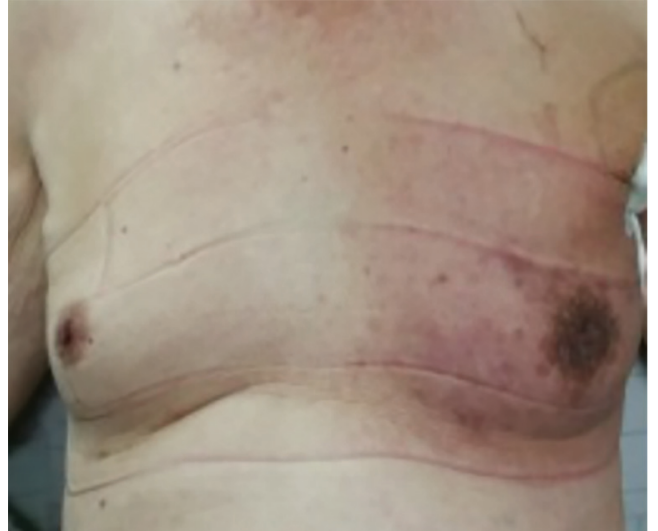

Figure 1 The skin of the left chest wall was prominently red and swollen.

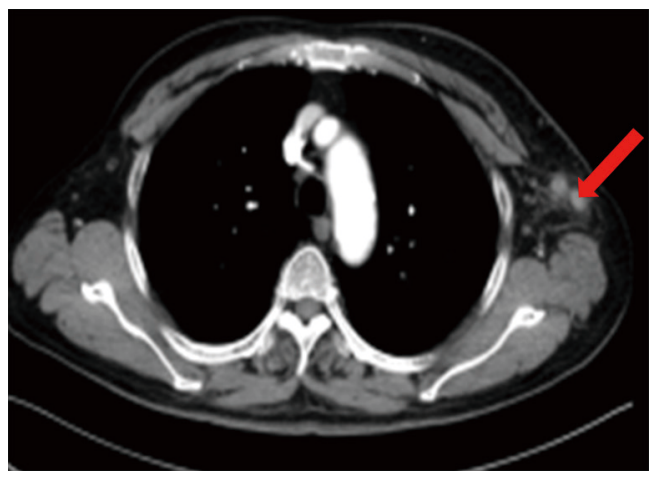

Figure 2 A CT image showing the left enlarging lymph nodes (see the arrowhead).

appear to have experienced any antecedent trauma or infection, and did not present with any joint pain or other symptoms (e.g., fever, night sweat or weight loss). The patient had no history of drug addiction or chest radiation. He also had no family history of breast cancer or atopy. The patient had been previously treated with antibiotics under the guidance of doctors at a local hospital, but that treatment was ineffective.

The results of a physical examination showed that most of the patient's left chest wall skin was red and there was swelling, especially around the areola, without ulceration or pustules (see Figure 1). There was no nipple retraction or discharge. The patient also presented with scatted nodules that could be touched on his left breast. The ipsilateral lymph node was palpable. Conversely, the right breast and axilla were normal. A computed tomography (CT) scan of the thorax (see Figure 2) showed a nodule in the left breast with an indistinct margin and mild enhancement. The left axillary lymph nodes were increased in terms of volume and quantity. An ultrasound (see Figure 3) revealed that the skin layer of the left chest had thickened and now measured $4 \mathrm{~mm}$. There was a $0.8 \mathrm{~cm} \times 0.7 \mathrm{~cm} \times 0.8 \mathrm{~cm}$ irregular, ill-defined hypoechoic mass with vascularity in the left breast subareolar region, accompanied by left axillary and supraclavicular lymphadenopathy.

Laboratory tests, including a complete blood cell count, erythrocyte sedimentation rate test, a C-reactive protein test, a coagulation panel and a serum test (for antinuclear antibodies, the rheumatoid factor, cytoplasmic antineutrophil, cytoplasmic antibodies, and immunoglobulin G), were conducted to exclude other possibilities. All the results were within normal limits. Additionally, the patient had acquired neither an immunosuppression nor a hematologic disorder. The function of his liver and kidney was also normal. However, the levels of hormones in serum, including the luteinizing hormone, follicle-stimulating hormone and progesterone, were higher than normal.

The patient underwent an ultrasound-guided core needle biopsy $(\mathrm{CNB})$ of his left breast mass, and an ultrasoundguided fine needle aspiration cytologic test of an enlarged left axillary lymph node. The results confirmed that the patient had chronic inflammation of the lymphocytes and plasma cells. Further, the lymph nodes appeared to display reactive hyperplasia. Next, a left breast mastectomy was performed. A specimen (measuring $13 \mathrm{~cm} \times 10 \mathrm{~cm} \times$ $2.5 \mathrm{~cm}$ ) was removed and sectioned. The histopathologic results showed that there were infiltrations of lymphocytes and plasma cells in the dermis (see Figure 4). This was consistent with the symptoms characteristic of PCM; however, lymphoma could not be ruled out. Thus, the immunohistochemical staining of the lymphocytes in the lesion was preformed to test the key marker of lymphoma. We found that the lymphocytes CD20, CD3, and CD38 were positive, but IgG4 was negative. Additionally, the results of in-situ hybridization revealed that EBER1/2 was negative. Thus, a possible diagnosis of lymphoma was excluded. Written informed consent was obtained from the patient for publication of this manuscript and any accompanying images. All procedures performed in studies involving human participants were in accordance with the ethical standards of the institutional and/or national research committee(s) and with the Helsinki Declaration (as revised in 2013). 

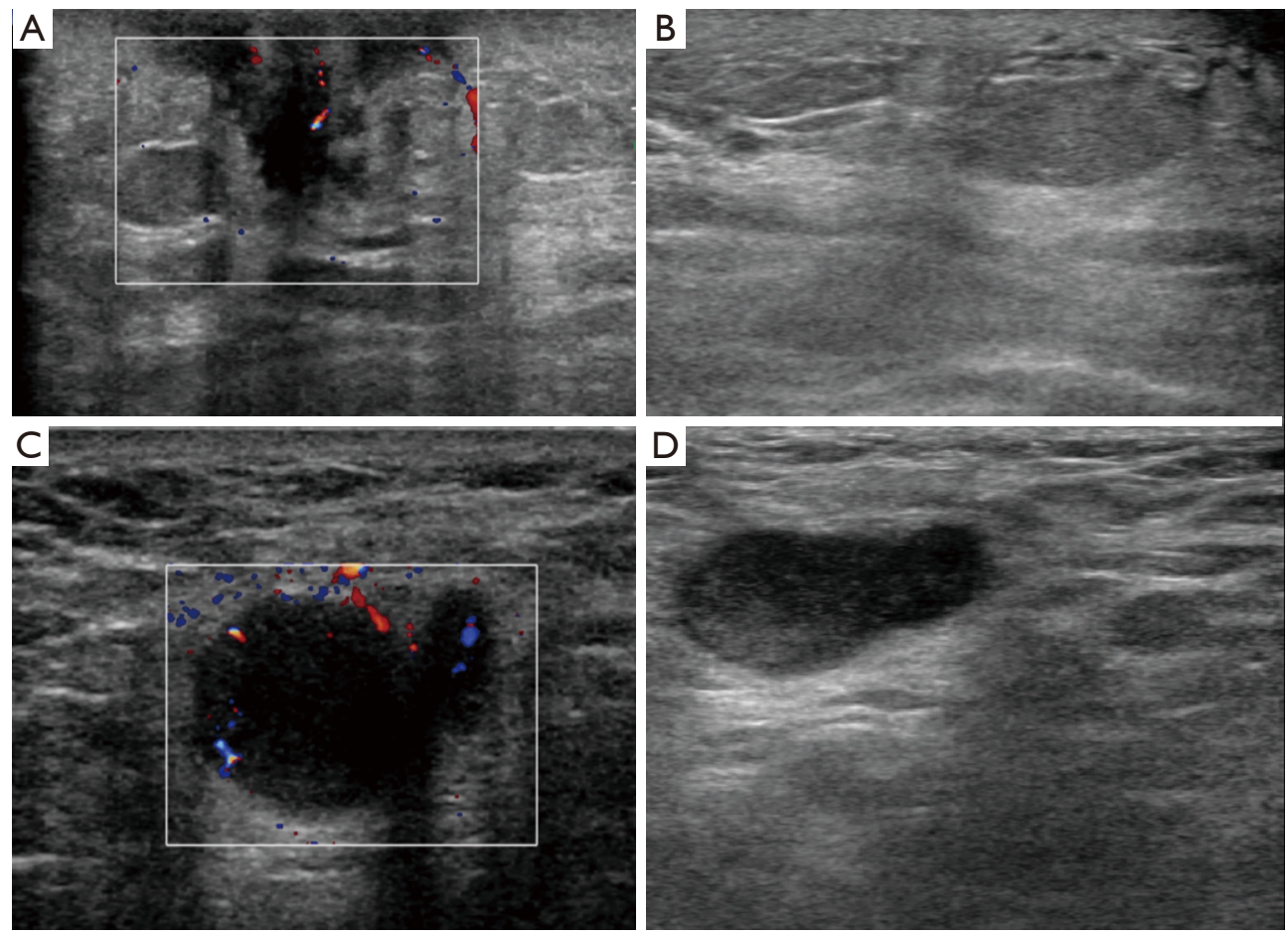

Figure 3 Ultrasound scans of the patient's left breast revealed an $8 \mathrm{~mm} \times 7 \mathrm{~mm} \times 8 \mathrm{~mm}$, lobulated, irregularly shaped, hypoechoic subareolar area and skin thickening on the left chest wall, and showed that the left axillary lymph node was large and abnormal in structure. (A) Ultrasound scans of the patient's left breast revealed an $8 \mathrm{~mm} \times 7 \mathrm{~mm} \times 8 \mathrm{~mm}$, lobulated, irregularly shaped, hypoechoic subareolar area; (B) an ultrasound revealed that the skin layer of the left chest had thickened; (C and D) an ultrasound revealed that the left axillary lymph node was large and abnormal in structure.

\section{Discussion}

PCM is a relatively common benign disease of the breast in non-puerperal, young and middle-aged women; however, it is very rare in men. To date, only 16 male cases appear to have been reported in English-language literature (3). This appears to be the first case in which the initial clinical signs included red, itchy and swollen skin, and an axillary mass. The etiology and pathogenesis of PCM are unknown. Multifarious factors, such as a hormonal imbalance, smoking, and autoimmunity, are considered possible causes of the disease. Many researchers have suggested that IL6/STAT3 signaling $(4,5)$, which may be involved in the pathogenesis, is activated in PCM. The common clinical manifestation of PCM includes a retro-areolar lump with an inverted nipple and secretions, ductal ectasia, and incidental mastodynia (6).

The results of mammography and ultrasound screenings for non-puerperal mastitis are non-specific (7). Mammography screenings often show focal/diffuse asymmetrical density, architectural disorder, or ill-defined masses. Occasionally, a mammography screening may show a normal parenchymal pattern. Ultrasonography screenings can reveal lesions that are irregular, heterogeneous, hypoechoic, and mass-like. As was also observed in our patient, some patients present with skin thickening in the left chest wall, hypoechogenicity in the sub-areola, and an enlarged ipsilateral lymph node. Ahistologic analysis is necessary for a diagnosis of PCM. The histopathologic characteristics of PCM include inflammatory infiltrate with lymphocytes, plasma cells and ductal dilatation. In some cases, abscess evolution may be observed (3).

It is essential that carcinoma, lymphoma, and other lesions that may be similar to PCM are excluded. For this patient, a clinical differential diagnosis excluded lymphoma, IBC, and IgG4-related sclerosing disease. Janes et al. (8) found that a breast CNB plays a significant role in the diagnosis of unilateral breast lumps in men. Asadi et al. (9) noted that a CNB is not reliable for the diagnosis of some PCMs, as PCM is a difficult to differentiate cancer. It is 

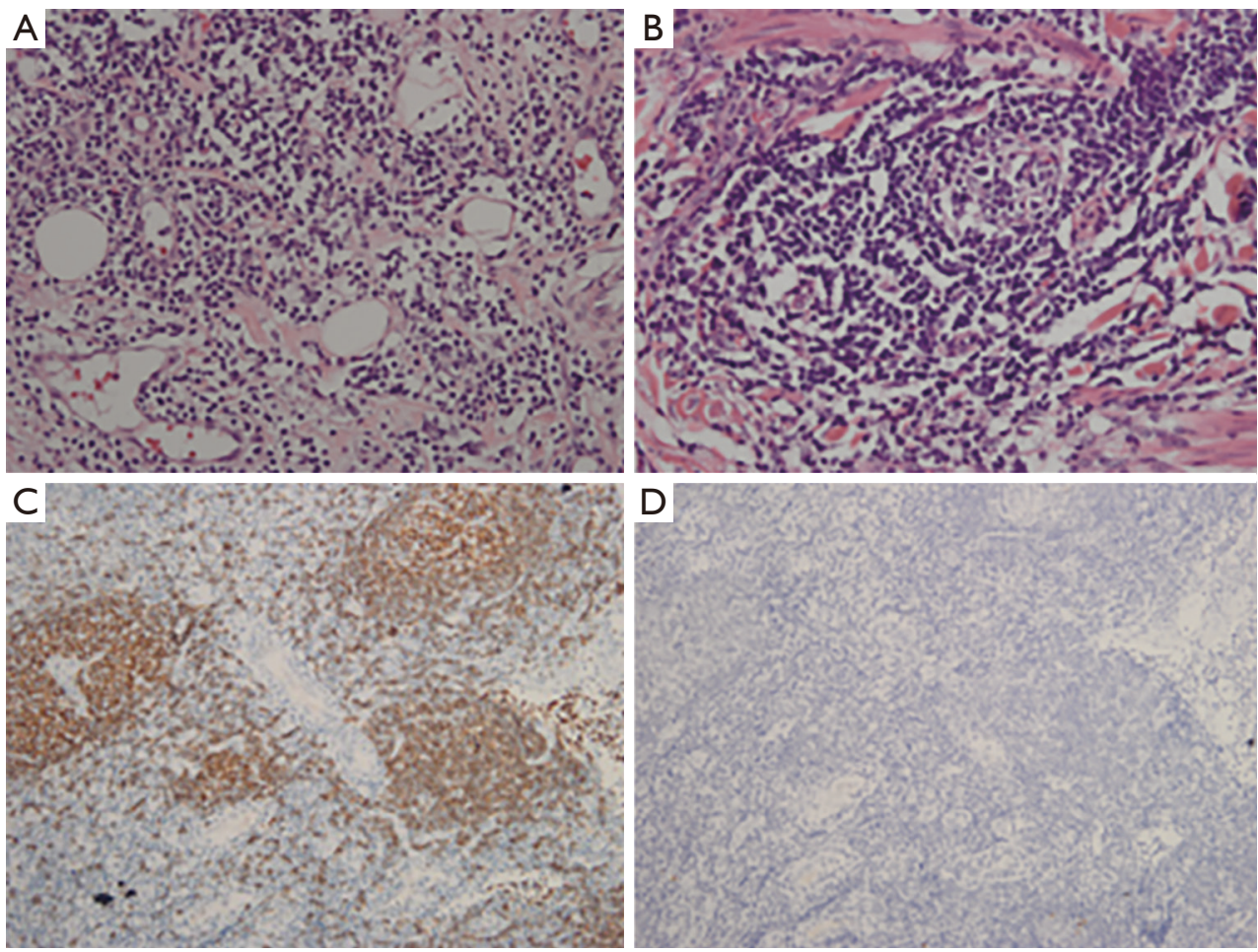

Figure 4 Plasma cell mastitis. CNB $(\times 200)$ and an excisional examination $(\times 400)$ H\&E-stained section showing a large number of lymphocytes and plasma cells infiltrating the interstitial, glial deposition. lymphoid hematopoietic tumors could not be ruled out. The CD20, CD3, and CD38 immunostaining was positive, and the IgG 4 immunostaining was negative. (A) H\&E staining; magnification, $\times 400$; (B) H\&E staining; magnification, $\times 400$; (C) by using immunohistochemistry, the expression of CD20 is positive; magnification, $\times 200$; (D) by using immunohistochemistry, the expression of IgG4 is negative; magnification, $\times 200$. CNB, core needle biopsy; H\&E staining, hematoxylin-eosin.

different to rule out carcinoma; however, an excision biopsy is mandatory.

As a rare and aggressive disease, IBC leads to $7-10 \%$ of breast cancer-related mortality and has a morbidity rate of $2-4 \%(10)$. This disease is diagnosed by the following clinical features: the involvement of $\geq 30 \%$ of the affected breast and/or skin, architectural changes (such as erythema and peau d'orange-like changes), and the confirmation of invasive breast cancer either by a skin or parenchymal biopsy of the breast. The above-mentioned changes should occur within 3-6 months of their first appearance (10). Given that the patient in this study presented with erythema and edema in the left chest wall within 6 months, and his palpable axillary lymph node, the disease could be considered IBC.

IgG4-related sclerosing disease is a systemic autoimmune entity. Typically, patients with the disease have a raised serum concentration of IgG4 and mass-forming lesions with fibrosis and dense lymphoplasmacytic infiltrate with increased IgG4-positive plasma cells. The breast is one of the organs affected by it. Vitkovski et al. (11) reported the first male case in 2017. The results of a laboratory test and immunohistochemical staining (see Figure 4D) helped us to exclude the possibility of the disease.

In the treatment of PCM, general antibiotics therapy is often unsuccessful; this was observed to be the case in our patient. At present, high-dose glucocorticoid therapy $(0.5-1 \mathrm{mg} / \mathrm{kg} /$ day) has been referenced as the standard treatment that can effectively control benign inflammatory breast disease. However, if the dose is decreased, PCM commonly recurs (6). Akbulut et al. (12) studied 202 patients with idiopathic granulomatous mastitis, and found that methotrexate had a good efficiency. Faccin et al. (13) reported that a tumor necrosis factor (TNF) antagonist can improve the clinical manifestations of PCM in some cases. Faccin et al. further conjectured that a TNF antagonist 
might have a glucocorticoid-sparing effect. Given the high recurrence rate in cases in which a conservative treatment is adopted, surgical excision, including lesion excision and mastectomy, is the most common choice for surgeons and patients. Our patient underwent a mastectomy, and was very satisfied with the management and outcome. There was no recurrence at the follow-up treatment 1 year later. $\mathrm{Wu}$ et al. (14) have advocated the use of fiberoptic ductoscopy plus with ultrasound-guided minimally invasive drainage for PCM, noting that this treatment not only had a high cure rate and low recurrence rate, but also enabled breast integrity to be maintained. Ming et al. (6) analyzed 91 cases, and suggested that early and complete focused excision and nipple retraction correction are effective treatment methods for PCM. Given the high recurrence of PCM and the breast abnormality, the treatment of PCM represents an enormous challenge that requires further exploration.

\section{Conclusions}

In this paper, a case of an atypical PCM was reported. Some of the clinical findings of PCM are similar to those of IBC. For example, the initial symptoms of our patient included skin redness and edema associated with pruritus, which is rare in clinical research. Ultimately, laboratory tests and immunohistochemical staining were conducted that confirmed that this male patient suffered from PCM. The etiology and optional treatment of PCM should be subject to further research.

\section{Acknowledgments}

The authors gratefully acknowledge all of the staff in the Department of Breast Surgery, West China Hospital, who generously provided assistance in the collection of data throughout the duration of the study.

Funding: None.

\section{Footnote}

Reporting Checklist: The authors have completed the CARE reporting checklist. Available at http://dx.doi.org/10.21037/ gs-21-40

Conflicts of Interest: All authors have completed the ICMJE uniform disclosure form (available at http://dx.doi. org/10.21037/gs-21-40). The authors have no conflicts of interest to declare.
Ethical Statement: The authors are accountable for all aspects of the work in ensuring that questions related to the accuracy or integrity of any part of the work are appropriately investigated and resolved. Written informed consent was obtained from the patient for publication of this manuscript and any accompanying images. All procedures performed in studies involving human participants were in accordance with the ethical standards of the institutional and/or national research committee(s) and with the Helsinki Declaration (as revised in 2013).

Open Access Statement: This is an Open Access article distributed in accordance with the Creative Commons Attribution-NonCommercial-NoDerivs 4.0 International License (CC BY-NC-ND 4.0), which permits the noncommercial replication and distribution of the article with the strict proviso that no changes or edits are made and the original work is properly cited (including links to both the formal publication through the relevant DOI and the license). See: https://creativecommons.org/licenses/by-nc-nd/4.0/.

\section{References}

1. Giordano SH. A review of the diagnosis and management of male breast cancer. Oncologist 2005;10:471-9.

2. Nagaratnam N, Kannangara CS. Plasma cells mastitis. Postgrad Med J 1958;34:654-5.

3. Palmieri A, D'Orazi V, Martino G, et al. Plasma cell mastitis in men: a single-center experience and review of the literature. In Vivo 2016;30:727-32.

4. Liu Y, Zhang J, Zhou YH, et al. IL-6/STAT3 signaling pathway is activated in plasma cell mastitis. Int J Clin Exp Pathol 2015;8:12541-8.

5. Liu Y, Zhang J, Zhou YH, et al. Activation of the IL-6/ JAK2/STAT3 pathway induces plasma cell mastitis in mice. Cytokine 2018;110:150-8.

6. Ming J, Meng G, Yuan Q, et al. Clinical characteristics and surgical modality of plasma cell mastitis: analysis of 91 cases. Am Surg 2013;79:54-60.

7. Tan H, Li R, Peng W, et al. Radiological and clinical features of adult non-puerperal mastitis. Br J Radiol 2013;86:20120657.

8. Janes SE, Lengyel JA, Singh S, et al. Needle core biopsy for the assessment of unilateral breast masses in men. Breast 2006;15:273-5.

9. Asadi M, Nouri M, Jangjoo A, et al. Core needle biopsy (CNB) to differentiate plasma cell mastitis from breast cancer. Eur J Surg Oncol 2012;38:817-8. 
10. Fouad TM, Barrera AMG, Reuben JM, et al. Inflammatory breast cancer: a proposed conceptual shift in the UICC-AJCC TNM staging system. Lancet Oncol 2017;18:e228-32.

11. Vitkovski T, Marder GS, Filardi DA. et al. IgG4-Related Sclerosing Disease of the Breast in a Male Patient. Int J Surg Pathol 2017;25:711-5.

12. Akbulut S, Yilmaz D, Bakir S, et al. Methotrexate in the management of idiopathic granulomatous mastitis: review of 108 published cases and report of four cases. Breast J 2011;17:661-8.

Cite this article as: Long Q, Fan Y, Zhang J, Li H, Lv Q. Pruritic breast mass with palpable lymph nodes in a male patient: a case report. Gland Surg 2021;10(2):826-831. doi: $10.21037 /$ gs-21-40
13. Faccin M, Caillot O, Levêque J, et al. Plasma cell mastitis in women with rheumatoid arthritis treated with TNF antagonists: Report of 2 cases. Joint Bone Spine 2016;83:593-4.

14. Wu HL, Yu JJ, Yu SL, et al. Clinical efficacy of fiberoptic ductoscopy in combination with ultrasound-guided minimally invasive surgery in treatment of plasma cell mastitis. Clin Exp Obstet Gynecol 2016;43:742-6.

(English Language Editor: L. Huleatt) 